\title{
Dietary carbohydrate restriction augments weight loss-induced improvements in glycaemic control and liver fat in individuals with type 2 diabetes: a randomised controlled trial
}

\author{
Mads N. Thomsen ${ }^{1}$ (D) Mads J. Skytte ${ }^{1}$ (D) Amirsalar Samkani ${ }^{1}$ (D) $\cdot$ Martin H. Carl ${ }^{1} \cdot$ Philip Weber $^{1} \cdot$ Arne Astrup $^{2}$ (D) \\ Elizaveta Chabanova $^{3} \cdot$ Mogens Fenger $^{4}$ (D) Jan Frystyk ${ }^{5,6}$ (D) Bolette Hartmann ${ }^{7,8}$ (D) Jens J. Holst ${ }^{7,8}$ (D) \\ Thomas M. Larsen $^{2}$ (D) - Sten Madsbad ${ }^{9}$ (D) - Faidon Magkos ${ }^{2}$ (D) - Henrik S. Thomsen ${ }^{3}$ (D) Steen B. Haugaard ${ }^{1}$ (D) \\ Thure Krarup $^{1,2}$ (D)
}

Received: 25 May 2021 / Accepted: 29 September 2021 / Published online: 7 January 2022

(C) The Author(s), under exclusive licence to Springer-Verlag GmbH Germany, part of Springer Nature 2022

\begin{abstract}
Aims/hypothesis Lifestyle modification and weight loss are cornerstones of type 2 diabetes management. However, carbohydrate restriction may have weight-independent beneficial effects on glycaemic control. This has been difficult to demonstrate because low-carbohydrate diets readily decrease body weight. We hypothesised that carbohydrate restriction enhances the beneficial metabolic effects of weight loss in type 2 diabetes.

Methods This open-label, parallel RCT included adults with type 2 diabetes, $\mathrm{HbA}_{1 \mathrm{c}} 48-97 \mathrm{mmol} / \mathrm{mol}(6.5-11 \%)$, BMI $>25 \mathrm{~kg} / \mathrm{m}^{2}$, eGFR $>30 \mathrm{ml} \mathrm{min}{ }^{-1}[1.73 \mathrm{~m}]^{-2}$ and glucose-lowering therapy restricted to metformin or dipeptidyl peptidase- 4 inhibitors. Participants were randomised by a third party and assigned to 6 weeks of energy restriction (all foods were provided) aiming at $\sim 6 \%$ weight loss with either a carbohydrate-reduced high-protein diet (CRHP, percentage of total energy intake [E\%]: $\mathrm{CH} 30 / \mathrm{P} 30 / \mathrm{F} 40)$ or a conventional diabetes diet (CD, E\%: $\mathrm{CH} 50 / \mathrm{P} 17 / \mathrm{F} 33)$. Fasting blood samples, continuous glucose monitoring and magnetic resonance spectroscopy were used to assess glycaemic control, lipid metabolism and intrahepatic fat. Change in $\mathrm{HbA}_{1 \mathrm{c}}$ was the primary outcome; changes in circulating and intrahepatic triacylglycerol were secondary outcomes. Data were collected at Copenhagen University Hospital (Bispebjerg and Herlev).

Results Seventy-two adults (CD 36, CRHP 36, all white, 38 male sex) with type 2 diabetes (mean duration 8 years, mean $\mathrm{HbA}_{1 \mathrm{c}} 57 \mathrm{mmol} / \mathrm{mol}$ [7.4\%]) and mean BMI of $33 \mathrm{~kg} / \mathrm{m}^{2}$ were enrolled, of which 67 (CD 33, CRHP 34) completed the study. Body weight decreased by $5.8 \mathrm{~kg}(5.9 \%)$ in both groups after 6 weeks. Compared with the CD diet, the CRHP diet further reduced $\mathrm{HbA}_{1 \mathrm{c}}$ (mean $\left.[95 \% \mathrm{CI}]-1.9[-3.5,-0.3] \mathrm{mmol} / \mathrm{mol}[-0.18(-0.32,-0.03) \%], p=0.018\right)$ and diurnal mean glucose (mean $[95 \% \mathrm{CI}]-0.8[-1.2,-0.4] \mathrm{mmol} / \mathrm{l}, p<0.001$ ), stabilised glucose excursions by reducing
\end{abstract}

Steen B. Haugaard and Thure Krarup share senior authorship.

Mads N. Thomsen

mads.norvin.thomsen@regionh.dk

1 Department of Endocrinology, Copenhagen University Hospital Bispebjerg, Copenhagen, Denmark

2 Department of Nutrition, Exercise and Sports, University of Copenhagen, Copenhagen, Denmark

3 Department of Radiology, Copenhagen University Hospital Herlev, Copenhagen, Denmark

4 Department of Clinical Biochemistry, Copenhagen University Hospital Hvidovre, Copenhagen, Denmark
5 Department of Endocrinology, Odense University Hospital, Odense, Denmark

6 Department of Clinical Medicine, Aarhus University, Aarhus, Denmark

7 Novo Nordisk Foundation Center for Basic Metabolic Research, University of Copenhagen, Copenhagen, Denmark

8 Department of Biomedical Sciences, University of Copenhagen, Copenhagen, Denmark

9 Department of Endocrinology, Copenhagen University Hospital Hvidovre, Copenhagen, Denmark 


\section{Research in context}

\section{What is already known about this subject?}

- Nutritional recommendations for the management of type 2 diabetes and obesity focus on weight loss but no ideal macronutrient distribution has been established

- Carbohydrate-restricted diets may improve glycaemic control in individuals with type 2 diabetes but the improvement often coincides with reductions in body weight, making interpretation difficult

\section{What is the key question?}

- Does a carbohydrate-reduced high-protein (CRHP) diet improve glycaemic control, liver fat and circulating triacylglycerol compared with a conventional diabetes diet after the same weight loss?

\section{What are the new findings?}

- A CRHP diet (fully provided as part of the study) for 6 weeks improves $\mathrm{HbA}_{1 c}$, diurnal mean glucose, glycaemic variability, liver fat and fasting triacylglycerol concentration beyond the effects of weight loss itself

\section{How might this impact on clinical practice in the foreseeable future?}

- Moderate carbohydrate restriction may be used in addition to weight loss in ameliorating hyperglycaemia and liver fat accumulation in individuals with type 2 diabetes and obesity and should be considered independent of energy restriction when designing nutritional therapies

glucose CV (mean $[95 \% \mathrm{CI}]-4.1[-5.9,-2.2] \%, p<0.001$ ), and augmented the reductions in fasting triacylglycerol concentration (by mean $[95 \% \mathrm{CI}]-18[-29,-6] \%, p<0.01$ ) and liver fat content (by mean $[95 \% \mathrm{CI}]-26[-45,0] \%, p$ $=0.051$ ). However, pancreatic fat content was decreased to a lesser extent by the CRHP than the CD diet (mean [95\% $\mathrm{CI}] 33[7,65] \%, p=0.010$ ). Fasting glucose, insulin, HOMA2-IR and cholesterol concentrations (total, LDL and HDL) were reduced significantly and similarly by both diets.

Conclusions/interpretation Moderate carbohydrate restriction for 6 weeks modestly improved glycaemic control, and decreased circulating and intrahepatic triacylglycerol levels beyond the effects of weight loss itself compared with a CD diet in individuals with type 2 diabetes. Concurrent differences in protein and fat intakes, and the quality of dietary macronutrients, may have contributed to these results and should be explored in future studies.

Trial registration ClinicalTrials.gov NCT03814694.

Funding The study was funded by Arla Foods amba, The Danish Dairy Research Foundation, and Copenhagen University Hospital Bispebjerg Frederiksberg.

Keywords Carbohydrate restriction $\cdot$ Dietary intervention $\cdot$ Dyslipidaemia $\cdot$ Glycaemic control $\cdot$ Low-energy diet $\cdot$ Non-alcoholic fatty liver disease $\cdot$ Nutritional therapy $\cdot$ Obesity $\cdot$ Type 2 diabetes mellitus $\cdot$ Weight loss

$\begin{array}{ll}\text { Abbreviations } \\ \text { Apo } & \text { Apolipoprotein } \\ \text { CD } & \text { Conventional diabetes } \\ \text { CGM } & \text { Continuous glucose monitoring } \\ \text { CRHP } & \text { Carbohydrate-reduced high-protein } \\ \text { DNL } & \text { De novo lipogenesis } \\ \text { DPP-4 } & \text { Dipeptidyl peptidase-4 } \\ \text { E\% } & \text { Percentage of total energy intake } \\ \text { MR } & \text { Magnetic resonance } \\ \text { SAT } & \text { Subcutaneous adipose tissue } \\ \text { TEE } & \text { Total energy expenditure } \\ \text { VAT } & \text { Visceral adipose tissue }\end{array}$

\section{Abbreviations}

CD Conventional diabetes

CGM Continuous glucose monitoring

CRHP Carbohydrate-reduced high-protein

vo lipogenesis

Deptidyl peptidase-4

E\% Percentage of total energy intake

agnetic resonance

TEE Total energy expenditure

VAT Visceral adipose tissue

\section{Introduction}

Most people with type 2 diabetes and excess body weight have comorbidities such as hypertension, atherogenic dyslipidaemia and non-alcoholic fatty liver disease, contributing to increased disability and mortality [1]. Weight loss improves glycaemic control [2] and ameliorates abnormalities in lipid metabolism [3], which may attenuate risk of microvascular complications [4] and CVD [5], respectively, and may lead to remission of type 2 diabetes [2]. Therefore, lifestyle modification with emphasis on negative energy balance 
facilitating weight loss is a cornerstone in managing individuals with obesity and type 2 diabetes [6].

Traditionally, an energy-reduced diet rich in fibre, rich in low-glycaemic index carbohydrate and limited in fat (allowing $10-20 \%$ of total energy intake from protein) has been the recommended dietary approach in type 2 diabetes treatment [7]. Recently, the ADA concluded in a consensus statement that carbohydrate-restricted dietary regimens may provide viable treatment strategies in the management of type 2 diabetes, although no ideal macronutrient distribution has yet been established [8]. Results from a meta-analysis suggested that low-carbohydrate diets may improve glycaemic control and CVD risk in type 2 diabetes during at least 1 year, even without significant changes in body weight [9].

We recently demonstrated that a 6 week, weight-maintaining, carbohydrate-reduced high-protein (CRHP) diet improved glucose and lipid metabolism and reduced liver fat content compared with a conventional diabetes (CD) diet [10]. However, the clinical utility of low-carbohydrate diets beyond weight loss remains elusive, because these diets often readily decrease body weight despite considerable efforts to prevent this from happening [10,11], and current dietary regimens for weight loss typically utilise hypo-energetic diets with percentage of total energy intake $(\mathrm{E} \%)>50$ from carbohydrate $[2,12]$. Accordingly, we investigated the effects of matched weight loss, induced by 6 weeks of hypo-energetic CRHP vs CD feeding, on metabolic health including $\mathrm{HbA}_{1 \mathrm{c}}$ as the primary outcome and liver fat accumulation and circulating triacylglycerol as secondary outcomes in individuals with type 2 diabetes who were overweight or obese.

\section{Methods}

Study design and population The study was an open-label, parallel RCT with participants allocated in a 1:1 ratio to a hypo-energetic CD or CRHP diet for 6 weeks. Randomisation was performed in blocks of random size by an unrelated third party using $\mathrm{R}$ (extension package 'blockrand', Version 3.6.0; R, Boston, MA, USA) to generate a randomisation list.

Eligible participants were adults with BMI $>25 \mathrm{~kg} / \mathrm{m}^{2}$ diagnosed with type 2 diabetes, an $\mathrm{HbA}_{1 \mathrm{c}}$ of $48-97 \mathrm{mmol} / \mathrm{mol}$ (6.5-11.0\%), and who were receiving glucose-lowering therapy restricted to metformin or dipeptidyl peptidase-4 (DPP-4) inhibitors. Initial inclusion criteria for $\mathrm{HbA}_{1 \mathrm{c}}(>53 \mathrm{mmol} / \mathrm{mol}$ [7.0\%]) and BMI $\left(>27 \mathrm{~kg} / \mathrm{m}^{2}\right)$ were lowered prior to study initiation to facilitate recruitment. Exclusion criteria were as follows: previous cancer and heart failure; recent and ongoing ischaemic heart disease; severe disease of the liver, kidneys and alimentary tract; anaemia (blood haemoglobin $<7 \mathrm{mmol} / 1$ for men and $<6 \mathrm{mmol} / \mathrm{l}$ for women); renal dysfunction (eGFR $<30 \mathrm{ml} \mathrm{min}{ }^{-1}[1.73 \mathrm{~m}]^{-2}$ or urine albumin/creatinine ratio
$>300 \mathrm{mg} / \mathrm{g}$ ); inability to comply with study procedures; participation in other studies; lactation; pregnancy (or planning a pregnancy); treatment with systemic corticosteroids, sulfonylureas, sodium-glucose cotransporter 2 inhibitors or injectable hypoglycaemic medications; alcohol abuse, defined as consumption in excess of national recommendations ( $<168 \mathrm{~g} /$ week for men and $<84 \mathrm{~g} /$ week for women); and smoking during the past year. Participants were recruited from the Capital Region of Denmark from January 2019 to July 2020 and all visits took place at the Endocrinology Research Unit, Copenhagen University Hospital Bispebjerg, except for magnetic resonance (MR) assessments that were performed at Copenhagen University Hospital Herlev. Participants provided written, informed consent to the study protocol, which was approved by the Health Ethics Committee of Copenhagen and the Danish Data Protection Agency. The study is registered with ClinicalTrials.gov (registration no. NCT03814694) and was conducted in accordance with the Declaration of Helsinki.

Diet intervention The control CD diet and experimental CRHP diet differed in macronutrient composition, and respectively provided $50 \mathrm{E} \%$ and $30 \mathrm{E} \%$ as carbohydrate, $17 \mathrm{E} \%$ and $30 \mathrm{E} \%$ as protein, and $33 \mathrm{E} \%$ and $40 \mathrm{E} \%$ as fat (electronic supplementary material [ESM] Table 1). Study diets provided all daily energy and comprised seven different daily menus, with three main meals per day with or without two snacks (ESM Table 2). Raw food items and ingredients were used to prepare the various meals and snacks, which were cooked and provided to the participants ready for consumption. All meals were prepared in the metabolic kitchen at the Department of Nutrition, Exercise and Sports, University of Copenhagen and were distributed free-of-charge, twice weekly.

Participants were instructed to consume all meals and abstain from all alcohol- and energy-containing beverages as well as any food not provided by the investigators. Dietary adherence was evaluated twice weekly by using food records. In case of inability to consume all provided food (e.g. due to satiety or aversion), individual adjustments were made. Participants were asked prior to inclusion about food allergies, fastidiousness and dietary preferences and were excluded if their responses conflicted with compliance to the study diets.

Weight loss management Dietary regimens were designed to induce the same energy deficit during the first 5 weeks of the intervention period in order to achieve a $6 \%$ weight loss. During week 6, energy intake was adjusted upwards to ensure stabilisation at the new lower body weight and avoid a catabolic state during metabolic measurements. Weight loss management was individualised to each participant and occurred in three steps (ESM Table 3). First, total energy expenditure (TEE) was calculated by multiplying resting 
energy expenditure, estimated from the Mifflin-St Jeor equation [13], with a physical activity level of 1.6, reflecting a sedentary lifestyle. Second, the total energy equivalent of weight loss was calculated as $6 \%$ of baseline body weight $\times$ $27,000 \mathrm{~kJ} / \mathrm{kg}(6453 \mathrm{kcal} / \mathrm{kg})$, as we expected lost weight to comprise $\sim 75 \%$ adipose tissue and $\sim 25 \%$ lean mass [14]. Daily energy intake was then calculated as TEE reduced by an amount equal to the total energy equivalent of weight loss divided by the total duration of the weight-loss phase (i.e. 35 days). The calculated reduction was weighted with $80 \%$ initially and gradually increased to $120 \%$ at week 5 to allow for habituation. Third, the planned energy deficit was revisited twice weekly (based on body weight measurements) and adjusted as necessary by adding or subtracting CRHP or CD food items to ensure the targeted weight loss. Participants were instructed to maintain their habitual physical activity throughout the study. This was assessed at baseline and during week 5 of the intervention with the long form of the International Physical Activity Questionnaire (IPAQ) [15].

Anthropometry and body composition Body weight measurements were performed, after participants had fasted for $10 \mathrm{~h}$, on the same calibrated digital scale (Seca 701; Hamburg, Germany) at every visit. Assessment of body composition was performed at the beginning and the end of the study, after participants had fasted for $10 \mathrm{~h}$, by use of a Lunar iDXA dualenergy x-ray absorptiometry scanner (GE Healthcare, Waukesha, WI, USA).

Blood samples Blood samples were collected, after participants had fasted for $10 \mathrm{~h}$, at baseline and weeks 2, 4, 5 and 6 . Blood was collected in precooled EDTA-tubes and immediately centrifuged to separate plasma, and in tubes with clot activator, allowed to clot at room temperature, and centrifuged thereafter to separate serum. Measurement of $\mathrm{HbA}_{1 \mathrm{c}}$ was performed using HPLC on the Tosoh Automated Glycohemoglobin Analyzer HLC-723G8 (Tosoh Corporation, Japan). Plasma glucose was measured using the glucose oxidase method (YSI 2300 STAT Plus; YSI, OH, USA) and serum insulin and C-peptide were measured using electrochemiluminescence immunoassays (Cobas 6000 e601; Roche Diagnostics, Switzerland). Whole-body insulin resistance (HOMA2-IR) was calculated from fasting plasma glucose and insulin concentrations by using the updated HOMA2 calculator (version 2.2.3; https://www.dtu.ox.ac. uk/). Total cholesterol, LDL-cholesterol, HDL-cholesterol and triacylglycerol concentrations were determined in serum by enzymatic colorimetric methods, and apolipoprotein (apo) B, apoA-1 and cystatin $\mathrm{C}$ were measured by immunoturbidimetric assays (Cobas 6000 c501; Roche Diagnostics). Non-HDLcholesterol was calculated by subtracting HDL-cholesterol from total cholesterol. Cystatin $\mathrm{C}$ was used in the Chronic Kidney Disease Epidemiology Collaboration (CKD-EPI) equation from
2012 to calculate eGFR, by which renal function can be reliably assessed without confounding from changes in protein intake or body composition [16]. Serum total NEFA were quantified with an enzymatic colorimetric assay (Wako Chemicals, Germany).

MR MR measurements were conducted at the beginning and the end of the study using a 3.0 T Ingenia MRI system (Philips Healthcare, Best, the Netherlands) with a dStream torso coil. Single-voxel MR spectroscopy (Point RESolved Spectroscopy [PRESS]) was used to measure total hepatic and muscle fat fractions [17]. Chemical shift encoding-based water-fat imaging (mDixon) was used to measure pancreatic fat fraction, visceral adipose tissue (VAT), subcutaneous adipose tissue (SAT) and waist circumference. Pancreatic fat content was measured in duplicates, and the mean was used to minimise intra-observer variability. A single $10 \mathrm{~mm}$ thick transverse section acquired at the middle of L3 vertebra was used for quantification of VAT, SAT and waist circumference [18]. All MR data were analysed blinded to treatment.

Diurnal glucose profiles The diurnal glucose profiles were obtained from measurements over 7 days at baseline before the intervention and during the last week (week 6) of the intervention by continuous glucose monitoring (CGM) using the FreeStyle Libre Pro (Abbott Diabetes Care, Alameda, CA, USA), which provides subcutaneous interstitial glucose, blinded to the participant, every $15 \mathrm{~min}$. The glucose monitor was pre-calibrated and inserted a day prior to starting the measurement period. Data were evaluated as mean glucose, glucose CV, time-in-range (3.9-10 $\mathrm{mmol} / \mathrm{l})$ as well as time above/below range [19]. The variables were calculated for each $24 \mathrm{~h}$ period (midnight to midnight) and included in the analyses as the mean of monitored days.

Urine samples Twenty-four hour urine specimens were collected prior to the intervention and during week 5 of the intervention to determine the excretion of urea as a marker of protein intake and, thus, a marker of diet compliance [20], and albumin as a marker of renal function. Urea was analysed by kinetic testing with urease and glutamate and albumin by immunoturbidimetric analysis (Cobas c6000; Roche Diagnostics). Albumin values $<0.05 \mu \mathrm{mol} / 1$ (detection limit) were set to $0.025 \mu \mathrm{mol} / 1$.

Statistical analysis The primary outcome was the change in $\mathrm{HbA}_{1 \mathrm{c}}$ between diet groups; secondary outcomes were changes in hepatic fat content and serum triacylglycerol. The protocol included 47 additional pre-specified outcomes of which 16 are presented in this paper (ESM Table 4). On the basis of treatment effect differences between weight-maintaining CRHP and CD diets [10] and a significance level of 0.05 , we estimated that 21 participants in each group (total of 42 participants) would be required to detect an $\mathrm{HbA}_{1 \mathrm{c}}$ difference 


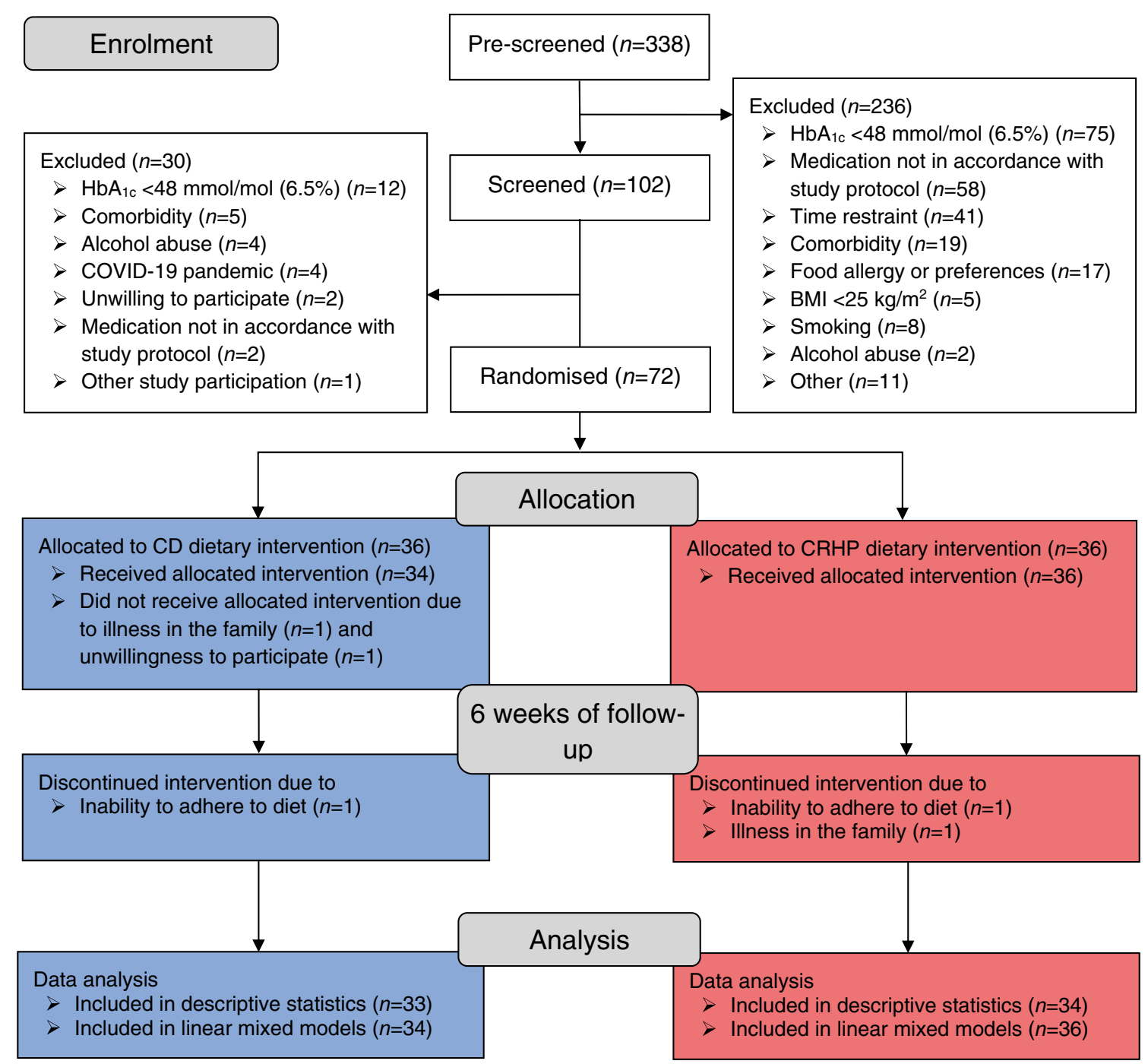

Fig. 1 Participant flow diagram. The median (25th, 75th percentile) time from randomisation to initiation of dietary intervention upon which participants were unblinded was $22(18,30)$ days for the CRHP diet and $22(19,31)$ days for the CD diet

of $5.4 \mathrm{mmol} / \mathrm{mol}(0.5 \%)$ between groups, with $90 \%$ power providing an SD of $5.2 \mathrm{mmol} / \mathrm{mol}(0.4 \%)$, whereas a $3.6 \%$ difference in liver fat (SD 4.8\%) could be detected with 29 participants per group (total of 58 participants) with $80 \%$ power. After accounting for a smaller than expected effect size and an anticipated $20 \%$ dropout rate, a final sample size of 80 participants was computed.

Treatment effects were evaluated using a constrained linear mixed model with inherent baseline adjustment and further assuming an unstructured covariance pattern to account for repeated measurements. Moreover, sensitivity analyses were conducted to further adjust for differences in sex, age, BMI, diabetes duration, and therapy with metformin and DPP-4 inhibitors. In accordance with an intention-to-treat principle, analyses included all available data and missing data were implicitly managed by maximum likelihood estimation, which is statistically optimal when missing data are assumed to be missing at random. Model assumptions were assessed from residual diagnostics and skewed outcomes were log-transformed prior to analysis. Between-diet comparisons are presented as estimated marginal means (95\% CIs), unless otherwise stated. Descriptive data are reported as mean \pm SD or median (25th, 75th percentile), depending on whether normal distribution assumptions were met or not, assessed visually and evaluated by the Shapiro-Wilks test.

Statistical tests were two-tailed and considered significant when $p<0.05$. All statistical analyses and graphics were performed using R (Version 3.6.0; R, Boston, MA, USA).

\section{Results}

Study flow and participants The recruitment process included 338 pre-screenings and 102 on-site screening visits; 72 participants were finally included in the study. Two participants 
Table 1 Baseline characteristics of study participants

\begin{tabular}{lll}
\hline Characteristic & CD diet & CRHP diet \\
\hline Participants/white, $n$ & $33 / 33$ & $34 / 34$ \\
Male/female sex, $n$ & $15 / 18$ & $20 / 14$ \\
Age, years & $67.0 \pm 8.8$ & $66.4 \pm 6.9$ \\
Duration of type 2 diabetes, years & $7.7(2.8,10.1)$ & $8.5(3.5,11.9)$ \\
$\mathrm{HbA}_{1 \mathrm{c}}, \mathrm{mmol} / \mathrm{mol}$ & $57.4 \pm 7.7$ & $57.6 \pm 8.4$ \\
$\mathrm{HbA}_{1 \mathrm{c}}, \%$ & $7.40 \pm 0.70$ & $7.42 \pm 0.77$ \\
$\mathrm{Body} \mathrm{weight,} \mathrm{kg}$ & $97.5 \pm 25.4$ & $98.0 \pm 14.2$ \\
$\mathrm{BMI}, \mathrm{kg} / \mathrm{m}^{2}$ & $33.2 \pm 5.1$ & $33.6 \pm 4.6$ \\
Estimated TEE, kJ/day & $10,881 \pm 2644$ & $11,100 \pm 1523$ \\
Medication use, $n(\%)^{\text {Glucose-lowering therapy }}$ & $21(64)$ & $26(76)$ \\
$\quad$ Lifestyle intervention only & $12(36)$ & $8(24)$ \\
$\quad$ Biguanides & $21(64)$ & $25(74)$ \\
$\quad$ DPP-4 inhibitors & $3(9)$ & $11(32)$ \\
Lipid-lowering therapy $^{\mathrm{a}}$ & $23(70)$ & $26(76)$ \\
Antihypertensive therapy & $26(79)$ & $29(85)$ \\
\hline
\end{tabular}

Data are presented as means \pm SD or medians (25th, 75 th percentiles) unless otherwise specified

${ }^{a}$ Lipid-lowering agents included statins (CD 23, CRHP 25) and one fibrate (CRHP)

${ }^{\mathrm{b}}$ Antihypertensive agents included ACE inhibitors (CD 9, CRHP 13), angiotensin receptor blockers (CD 16, CRHP 14), calcium channel blockers (CD 10, CRHP 15), diuretics (CD 14, CRHP 14) and adrenergic receptor blockers (CD 10, CRHP 7)

withdrew consent before the start of the study and three after starting the intervention, resulting in an attrition rate of $6.9 \%$ (CD 8.3\%, CRHP 5.6\%). Reasons for withdrawal were not related to adverse events or trial outcomes (Fig. 1). Because of restrictions due to the COVID-19 pandemic, 72 participants rather than the planned 80 were included in the study, with 67 completers securing statistical power to assess primary and secondary outcomes. Baseline characteristics were well balanced between groups (Table 1), except for a higher proportion of participants allocated to the CRHP diet group being male and being treated with DPP-4 inhibitors compared with the CD diet group. Metformin, DDP-4 inhibitors and all medications known to lower lipids and BP were stable for at least 2 months prior to study initiation and were kept unchanged throughout the study. Study results were not materially altered when adjusted for differences in diabetes medication and other relevant covariates (ESM Table 5).

Body weight, anthropometry and body composition Both interventions reduced body weight by a mean of $5.8 \mathrm{~kg}$ (Table 2) and this was successfully maintained during the last week (Fig. 2a). Waist circumference, body fat per cent, fat mass and fat-free mass decreased significantly in both groups without significant differences between diets. The energy intake gradually decreased from $\sim 7900 \mathrm{~kJ} /$ day during week 1 to $\sim 6300 \mathrm{~kJ} /$ day during week 5 of the intervention (i.e. before the weight maintenance week) (ESM Table 1), with no difference overall between diets $(19[-624,662] \mathrm{kJ} / \mathrm{day}, p=0.95)$.

Ectopic fat deposition Hepatic fat content was reduced significantly by $51 \%$ and $64 \%$ after the CD and CRHP diet, respectively, with the difference between groups reaching borderline significance $(-26[-45,0] \%, p=0.051)$ (Table 2). Pancreatic fat content decreased with both diets but to a significantly smaller extent by the CRHP diet when compared with the $\mathrm{CD}$ diet $(33[7,65] \%, p=0.010)$, while the muscle fat fraction did not change on either diet despite weight loss. Volumes of VAT and SAT were reduced significantly and similarly in the two diet groups.

Glycaemic control and metabolic variables $\mathrm{HbA}_{1 \mathrm{c}}$ was reduced after weight loss in both groups, and reductions were significantly greater with the CRHP than the CD diet $(-1.9$ $[-3.5,-0.3] \mathrm{mmol} / \mathrm{mol}(-0.18[-0.32,-0.03] \%), p=0.018)$ (Table 3, Fig. 2b). Fasting glucose (Fig. 2c), insulin (Fig. 2d), C-peptide and HOMA2-IR decreased to the same extent in both diet groups. Furthermore, when compared with the CD diet, the CRHP diet reduced fasting triacylglycerol concentration by $18(6,29) \%(p<0.01)$ (Fig. 2e). The changes in fasting NEFA, apoB, apoA-1, total cholesterol, LDL-cholesterol, HDL-cholesterol and non-HDL-cholesterol concentrations were similar between diets, and all lipid variables except for NEFA decreased significantly with weight loss (Table 3 and ESM Fig. 1).

CGM CGM data were available (median [25th, 75th percentile]) for $7(7,7)$ days both at baseline and at 6 weeks in both intervention groups. Both groups achieved improved glucose profiles after weight loss compared with the baseline habitual diet (Table 3). However, the reduction in diurnal mean glucose and glucose $\mathrm{CV}$ was greater after the CRHP diet than the CD diet by $-0.8(-1.2,-0.4) \mathrm{mmol} / \mathrm{l}(p<0.001)$ and $-4.1(-5.9,-2.2) \%$ $(p<0.001)$, respectively. Additionally, although the time-inrange did not significantly differ between groups, participants randomised to the CRHP diet spent significantly less time above $10.0 \mathrm{mmol} / \mathrm{l}(-3.5[-6.4,-0.6] \%, p=0.019)$ and significantly more time below $3.9 \mathrm{mmol} / 1$ (8.1 [2.4, $13.9] \%, p<0.01)$ throughout the day. The participants randomised to the CRHP diet were also more inclined to have CGM-determined hypoglycaemia (i.e. one CGM reading $<3.9 \mathrm{mmol} / \mathrm{l})(\mathrm{CD} 70 \%$, CRHP $82 \%$ ), including glucose values $<3.0 \mathrm{mmol} / 1$ (CD 9\%, CRHP 29\%) ( $p=$ 0.24 and $p=0.06$, respectively, by Fisher's Exact test), compared with those randomised to the CD diet.

Renal function and compliance measures No differences between diets were found in $\operatorname{eGFR}\left(5.3[2.9,13.5] \mathrm{ml} \mathrm{min}^{-1}\right.$ $\left.[1.73 \mathrm{~m}]^{-2}, p=0.21\right), 24 \mathrm{~h}$ total albumin excretion $(28[-4$, 
Table 2 Measures of anthropometry, body composition and fat distribution before and after matched weight loss by a CD or a CRHP diet in individuals with overweight or obesity and type 2 diabetes

\begin{tabular}{|c|c|c|c|c|c|c|c|c|}
\hline \multirow[t]{2}{*}{ Variable } & \multicolumn{3}{|l|}{$\mathrm{CD}$ diet } & \multicolumn{3}{|l|}{ CRHP diet } & \multicolumn{2}{|l|}{ Between diets } \\
\hline & Baseline & Treatment effect & $n$ & Baseline & Treatment effect & $n$ & Difference & $p$ value \\
\hline \multicolumn{9}{|l|}{ Anthropometry } \\
\hline Body weight, $\mathrm{kg}$ & $97.5 \pm 25.4$ & $-5.8 \pm 2.3^{\ddagger}$ & 33 & $98.0 \pm 14.2$ & $-5.8 \pm 1.8^{\ddagger}$ & 34 & $0.1(-0.6,0.7)$ & 0.83 \\
\hline BMI, $\mathrm{kg} / \mathrm{m}^{2}$ & $33.2 \pm 5.1$ & $-2.0 \pm 0.6^{\ddagger}$ & 33 & $33.6 \pm 4.6$ & $-2.0 \pm 0.6^{\ddagger}$ & 34 & $-0.0(-0.2,0.2)$ & 0.94 \\
\hline Waist circumference ${ }^{\mathrm{a}}, \mathrm{mm}$ & $1126 \pm 100$ & $-33 \pm 26^{+}$ & 30 & $1155 \pm 96$ & $-32 \pm 23^{\ddagger}$ & 34 & $-0.1(-12.4,12.2)$ & 0.99 \\
\hline \multicolumn{9}{|l|}{ Body composition ${ }^{\mathrm{a}}$} \\
\hline Fat-free mass, $\mathrm{kg}$ & $58.0 \pm 13.7$ & $-1.8 \pm 1.4$ & 32 & $58.8 \pm 9.9$ & $-1.4 \pm 1.6^{\ddagger}$ & 34 & $0.5(-0.2,1.1)$ & 0.18 \\
\hline Fat mass, $\mathrm{kg}$ & $40.0 \pm 13.2$ & $-3.9 \pm 2.0^{\ddagger}$ & 32 & $39.2 \pm 8.8$ & $-4.3 \pm 1.1^{\ddagger}$ & 34 & $-0.4(-1.1,0.3)$ & 0.24 \\
\hline Body fat fraction, $\%$ & $40.5 \pm 5.9$ & $-1.8 \pm 1.4^{\ddagger}$ & 32 & $39.9 \pm 6.2$ & $-2.3 \pm 1.1^{\ddagger}$ & 34 & $-0.5(-1.0,0.1)$ & 0.10 \\
\hline \multicolumn{9}{|l|}{ Fat distribution ${ }^{\mathrm{a}}$} \\
\hline Hepatic fat fraction, $\%$ & $6.2(3.2,13.1)$ & $-2.5(-5.8,-1.4)^{\ddagger}$ & 32 & $8.1(2.9,12.9)$ & $-5.4(-8.0,-1.3)^{\ddagger}$ & 34 & $-26(-45,0)^{\mathrm{b}}$ & 0.051 \\
\hline Pancreatic fat fraction, $\%$ & $11.8(6.5,21.6)$ & $-2.1(-3.5,-0.5)^{\ddagger}$ & 32 & $8.8(5.8,16.3)$ & $-1.1(-2.9,1.2)$ & 34 & $33(7,65)^{\mathrm{b}}$ & 0.010 \\
\hline Muscle fat fraction, $\%$ & $3.9(2.5,5.3)$ & $-0.8(-1.8,0.3)$ & 30 & $3.8(2.7,5.7)$ & $0.2(-1.6,1.1)$ & 34 & $15(-12,49)^{\mathrm{b}}$ & 0.31 \\
\hline VAT, $\mathrm{cm}^{3}$ & $293 \pm 88$ & $-41 \pm 37^{\ddagger}$ & 31 & $310 \pm 110$ & $-41 \pm 30^{\ddagger}$ & 34 & $0.9(-15.1,17.0)$ & 0.91 \\
\hline $\mathrm{SAT}, \mathrm{cm}^{3}$ & $303 \pm 122$ & $-20 \pm 28^{*}$ & 30 & $318 \pm 109$ & $-26 \pm 29$ & 34 & $-5.6(-19.4,8.2)$ & 0.42 \\
\hline
\end{tabular}

Data at baseline and changes from baseline are presented as means \pm SD or medians (25th, 75th percentiles); between-diet differences are estimated marginal means (95\% CIs), presented as absolute or relative differences (CRHP vs CD) for normally distributed or log-transformed data, respectively, and derived from constrained linear mixed models with inherent baseline adjustment using all available data

${ }^{a}$ Missing data were observed for measurements of waist circumference, fat distribution and body composition due to technical failures and unwillingness to undergo scanning procedure

${ }^{\mathrm{b}}$ Relative difference $(\%)$

$\$ p<0.001$ vs baseline

$69] \%, p=0.090)$, or albumin/creatinine ratio $(19[-10,58] \%$, $p=0.23$ ) (ESM Table 6). The CRHP diet increased daily urea excretion $(184$ [140, 229] mmol, $p<0.001)$ compared with the $\mathrm{CD}$ diet, consistent with a higher protein intake. Physical activity levels were not different between groups $(0[-27,37] \%, p=$ 0.99 ) and were unchanged from baseline. However, according to the scoring protocol, 14 inadequately or erroneously answered questionnaires were excluded from the analysis.

Adverse events Participants were asked about any adverse events at study visits at baseline and weeks 2, 4, 5 and 6 and instructed to contact the study personnel in case of serious adverse events. Thirteen participants (CD 5, CRHP 8) experienced symptoms of mild constipation, which were remedied by sufficient fluid intake and laxatives (CD 2, CRHP 7), including one severe case of obstipation (CRHP). Other symptoms were episodes of diarrhoea (CD 2, CRHP 2), episodes of dizziness (CD 1, CRHP 2) and increased tiredness or lack of energy (CD 0, CRHP 2). One participant (CRHP) experienced transient episodes of excessive sweating with increasing plasma creatinine, although no underlying medical cause could be identified. Despite the CGM-determined time spent below $3.9 \mathrm{mmol} / \mathrm{l}$ and the number of hypoglycaemia events being greater for participants on the CRHP diet, all hypoglycaemic events were asymptomatic and no episodes of severe hypoglycaemia as defined by the ADA [21] occurred.

\section{Discussion}

A clinically relevant weight loss induced by a CRHP diet resulted in greater improvements in $\mathrm{HbA}_{1 \mathrm{c}}$ and diurnal glucose profile when compared with a matched weight loss induced by a CD diet in individuals with type 2 diabetes. The CRHP diet also lowered fasting triacylglycerol concentrations and hepatic fat content to a greater extent than the CD diet. Nevertheless, weight loss per se was, as expected, highly effective in improving metabolic health.

Weight loss achieved by a standard diabetes diet has been shown to ameliorate abnormalities in glucose metabolism and decrease $\mathrm{HbA}_{1 \mathrm{c}}[2,12]$, particularly when at least $5 \%$ of initial body weight is lost [3]. While both diet groups in the present study achieved reductions in $\mathrm{HbA}_{1 \mathrm{c}}$ levels during weight loss, the CRHP diet reduced $\mathrm{HbA}_{1 \mathrm{c}}$ levels significantly more than the $\mathrm{CD}$ diet. This indicates that better glycaemic control can be achieved independent of weight loss by dietary carbohydrate restriction, although differences in other macronutrients (e.g. protein or monounsaturated fat) may have contributed [22]. Nevertheless, these improvements in $\mathrm{HbA}_{1 \mathrm{c}}$ may be 


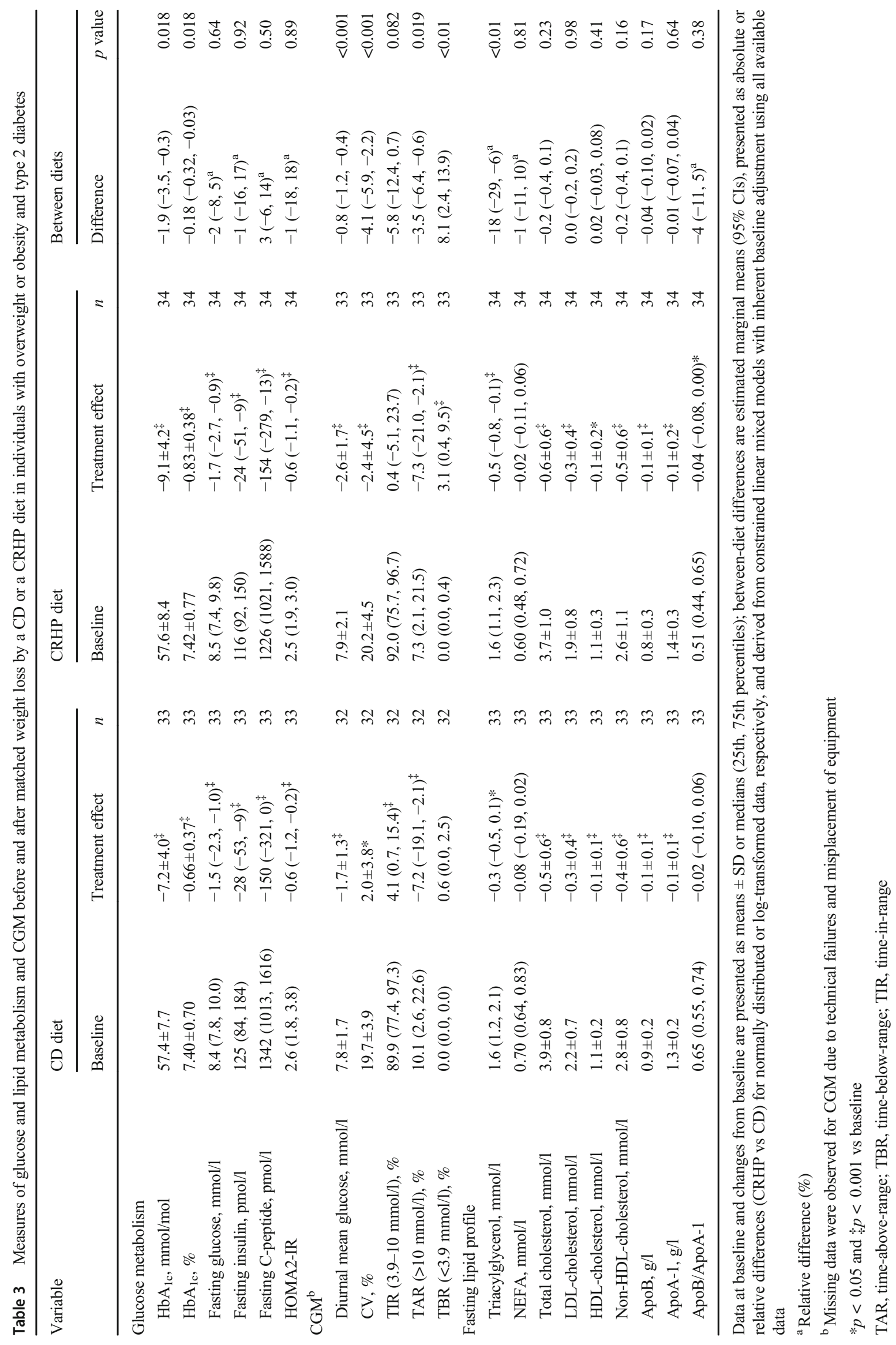



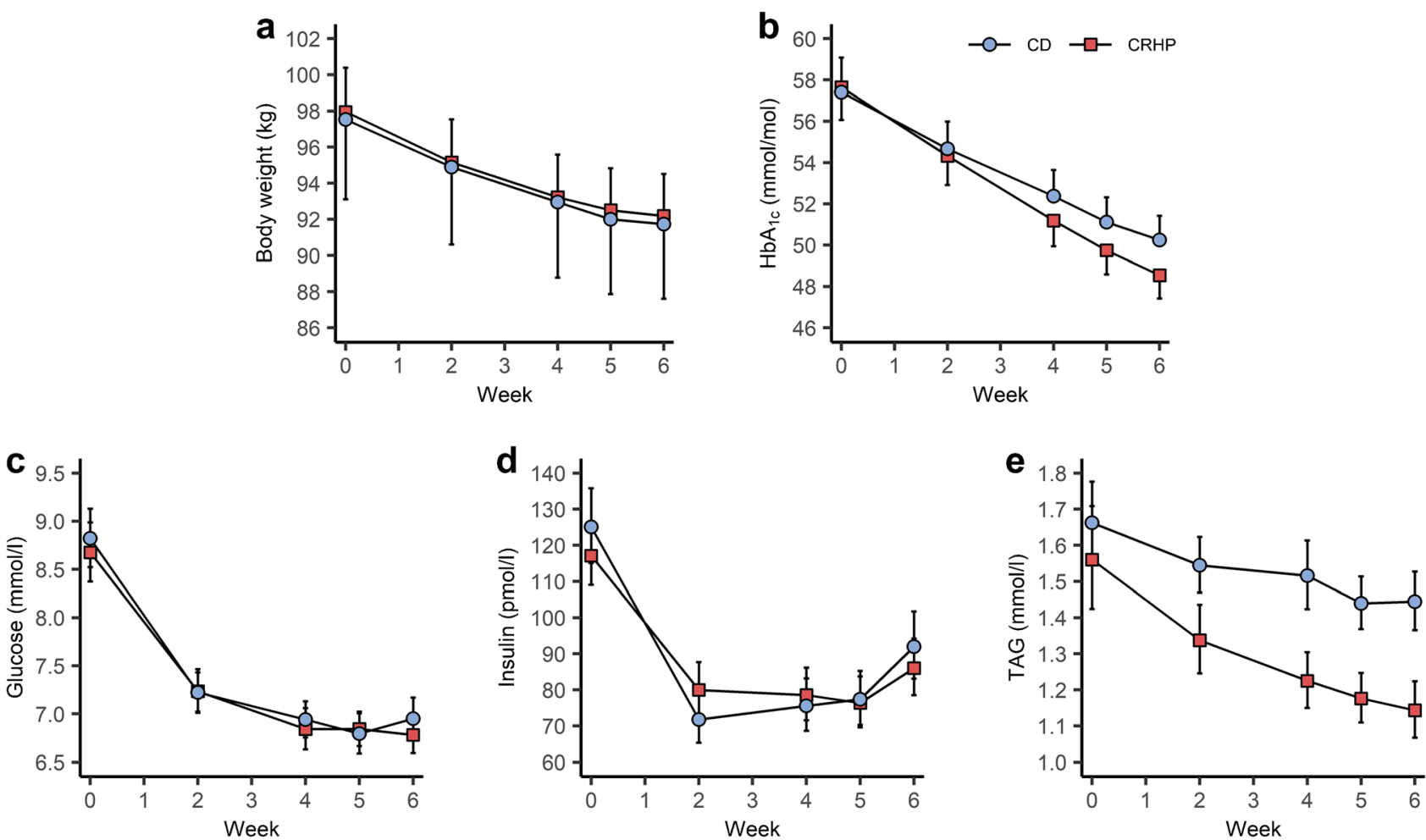

Fig. 2 Fasting measures of body weight $(p=0.83)(\mathbf{a}), \operatorname{HbA}_{1 \mathrm{c}}(p=0.018)(\mathbf{b})$, plasma glucose $(p=0.64)(\mathbf{c})$, serum insulin $(p=0.92)(\mathbf{d})$ and serum triacylglycerol (TAG) $(p<0.01)(\mathbf{e})$ during 6 weeks of a CRHP $(n=34)$ or CD $(n=33)$ diet. Data are presented as mean with SEM error bars, including back-transformed data in (c-e) following log-transformation. Changes from baseline to week 6 were evaluated between diets, and $p$ values for these differences are included

modest during energy restriction compared with iso-energetic feeding [10], underlying the primary importance of weight reduction over macronutrient composition. Iso-energetic meals with lower carbohydrate content acutely attenuate postprandial glucose and insulin excursions in type 2 diabetes, thus decreasing daily mean glucose [23, 24]. We made a similar observation during 6 weeks of a weight-maintaining CRHP diet that led to reduced $\mathrm{HbA}_{1 \mathrm{c}}$ compared with an isoenergetic $\mathrm{CD}$ diet [10]. Fasting glucose and insulin concentrations in the present study were equally reduced by the two diets. Diurnal mean glucose decreased significantly more with the CRHP diet, thus the greater reduction in $\mathrm{HbA}_{1 \mathrm{c}}$ has likely resulted from the persistent reductions in postprandial glucose excursions following the CRHP meals. Less carbohydrate being ingested with the CRHP meals compared with the CD meals may primarily drive the reductions in postprandial hyperglycaemia [23].

Diurnal glucose profiles are considered as supplementary to $\mathrm{HbA}_{1 \mathrm{c}}$ in evaluating the quality of glucose control; this includes time-in-range and glucose excursions as independent therapy targets $[19,21]$. Exacerbated glucose excursions measured as glycaemic variability (e.g. glucose CV) are progressively being considered as an independent CVD risk factor [25] and are readily attenuated in individuals with type 2 diabetes by carbohydrate restriction, even when in net energy balance [26]. We demonstrate here that reducing carbohydrate intake is beneficial even during hypo-energetic feeding and weight loss. The additional beneficial effects of carbohydrate restriction on hyperglycaemia and glycaemic variability could be of clinical importance and may persist even in combination with other lifestyle interventions [27].

Type 2 diabetes is often accompanied by excess accumulation of intrahepatic fat [28]. The pathogenesis of fatty liver disease involves abnormally increased hepatic de novo lipogenesis (DNL) in concert with elevated peripheral NEFA flux and a channelling of intrahepatic fatty acids towards storage [29]. Hyperinsulinaemia and hyperglycaemia augment hepatic DNL and contribute to liver fat accumulation and hypertriacylglycerolaemia [30], which can further elevate circulating NEFA levels through 'spill-over' during lipoprotein-bound triacylglycerol hydrolysis in the periphery [29]. We speculate that reversal of these processes by weight loss is responsible for much of the observed decreases in intrahepatic fat. Alcohol abstinence during the study may have exaggerated these decrements in both diet groups; however, a substantial contribution is unlikely given that none of the participants had a habitual alcohol abuse.

The greater decrease in liver fat with the CRHP diet than the $\mathrm{CD}$ diet may be responsible for the greater reduction in circulating triacylglycerol. In fact, we recently demonstrated 
that accumulation of liver fat in type 2 diabetes can be effectively reduced, in concert with fasting triacylglycerol levels, by iso-energetic substitution of carbohydrate by protein and fat during weight-stable conditions [10], and another study showed the same after iso-energetic substitution of carbohydrate by monounsaturated fat [31]. As such, our results may have been influenced by the higher intake of protein or monounsaturated fat in the CRHP diet [32]. Our finding is at odds with previous studies in overweight and obese individuals without diabetes, in which a moderate weight loss $(\sim 7 \%$ of baseline body weight) induced by low-carbohydrate and lowfat diets decreased liver fat to the same extent [33, 34]. However, it has been speculated that the presence of diabetes may fundamentally enhance the efficacy of carbohydrate restriction during both weight stability and weight loss [32].

It is difficult to evaluate the clinical relevance of the greater reduction in pancreatic fat content after the CD diet than after the CRHP diet. Studies evaluating intrapancreatic fat in relation to glucose metabolism and beta cell function in individuals with type 2 diabetes are scarce and provide conflicting data, possibly due to the largely heterogenous distribution of fat within the pancreas [35]. Nevertheless, reductions in pancreatic fat accumulation are thought to be important for recovery of pancreatic insulin secretion and, subsequently, for weight loss-induced remission of type 2 diabetes [36]. Our findings should thus be confirmed in future studies.

The long-term safety of carbohydrate restriction also needs to be considered as some people with type 2 diabetes are vulnerable to low-carbohydrate diet-induced hypoglycaemia [37]. The present study substantiates this concern, as participants randomised to the CRHP diet had significantly more CGM readings below $3.9 \mathrm{mmol} / \mathrm{l}$. Care should thus be taken, particularly in individuals already at risk of hypoglycaemia (e.g. those receiving insulin or sulfonylurea). Increased protein intake has also been scrutinised in diabetic kidney disease as a possible cause of further renal dysfunction [38], although individuals without overt kidney disease do not seem to be adversely affected [27].

Our study has several strengths including the standardised and tightly controlled intervention that involved full provision of the experimental diets and regular visits to maximise compliance, minimise attrition and achieve the same rate and amount of weight loss between groups. We found a high degree of adherence to the provided diets as indicated by the $65 \%$ greater $24 \mathrm{~h}$ urinary urea excretion on the CRHP diet (corresponding to the $76 \%$ greater dietary protein content) compared with the CD diet, even though the utility of this index during energy restriction and negative nitrogen balance may be limited [20]. Matching energy deficits and weight loss between groups is important because energy restriction and small differences in weight loss have been recognised as important confounders in trials of lowcarbohydrate diets [32, 39].

Our study is limited by the unblinded design and the imbalance of sex distribution and use of DPP-4 inhibitors between groups. We did not objectively evaluate physical activity, even though we used a self-reported tool that revealed no changes during the intervention and no differences between groups. The duration of our study was limited to 6 weeks, which is half the time required for $\mathrm{HbA}_{1 \mathrm{c}}$ reductions to achieve a new steady state. However, as $\mathrm{HbA}_{1 \mathrm{c}}$ levels decreased linearly with time during the CRHP diet (as shown previously during 10 weeks on a similar CRHP diet [40]) and the difference between our groups became progressively greater, the effect size following 2-3 months would likely have been greater than the one reported here after 6 weeks. This assertion is supported by the larger improvement in mean diurnal glucose, by $0.8 \mathrm{mmol} / 1(\sim 50 \%)$, after the CRHP diet than after the $\mathrm{CD}$ diet. Another limitation relates to multiple testing, which without adjustment increases the risk of falsepositive findings. Therefore, interpretation of the results for secondary and exploratory outcomes in the present study should be made with caution and our results should be confirmed in future studies. Finally, our study allowed the quality of dietary carbohydrate, protein and fat to vary naturally, reflecting the real foods used, and dietary components such as fibre (higher in the CD diet) and monounsaturated fat (higher in the CRHP diet) may have affected glycaemic control [22, 41, 42] and hepatic fat accumulation [31] independently of the macronutrient distribution.

In accordance with our results, some studies provide evidence of beneficial short-term effects of substituting carbohydrate with either protein or fat on glycaemic control and blood lipid profile in individuals with type 2 diabetes [9, 39]. However, meta-analyses are inconclusive regarding long-term benefits [9, 39] and, accordingly, conclusions from the recent consensus statement [8] on the efficacy of carbohydrate restriction remain ambiguous. Nonetheless, this may be related to decreasing dietary adherence over time [37, 39] or to heterogeneity among the included studies with respect to the composition and quality of dietary macronutrients, and differences in the amount of weight loss and concurrent exercise training. Adherence to low-carbohydrate diets may be particularly challenging when carbohydrate is severely restricted, whereas more moderate restrictions may be easier to comply with [37]. We have previously shown that individuals with type 2 diabetes can effectively migrate from full CRHP diet provision to a selfselected and self-prepared CRHP dietary pattern for at least 6 months in free-living conditions [43]. Before an optimal diet composition can be recommended, future studies should address long-term efficacy, feasibility and safety of different eating patterns, irrespective of concurrent lifestyle modification, as well as their additive or synergistic effects with weight loss [8].

In conclusion, a $6 \%$ weight loss induced by a CRHP diet over 6 weeks modestly improved $\mathrm{HbA}_{1 \mathrm{c}}$, diurnal 
glucose, circulating triacylglycerol and liver fat to a greater extent than the same amount of weight loss induced by a $\mathrm{CD}$ diet. These findings indicate that during matched weight loss, moderate carbohydrate restriction with subsequent increases in protein and fat can modestly improve metabolic health in individuals with obesity and type 2 diabetes, at least in the shortterm. This may be relevant when designing dietary strategies for the management of type 2 diabetes.

Supplementary Information The online version contains peer-reviewed but unedited supplementary material available at https://doi.org/10.1007/ s00125-021-05628-8.

Acknowledgements The authors are grateful for the participants' devoted efforts, which made this study possible. The authors thank the kitchen personnel at the Department of Nutrition, Exercise and Sports, University of Copenhagen. Preliminary results from the present study were presented at the 80th Scientific Session of the ADA, 12-16 June 2020 and at the 56th Annual Meeting of the EASD, 21-25 September 2020.

Data availability The datasets generated and analysed during the current study are available from the corresponding author on reasonable request and approval by the Danish Data Protection Agency.

Funding The study was funded by grants from Arla Foods amba, The Danish Dairy Research Foundation, and Copenhagen University Hospital Bispebjerg Frederiksberg. These study sponsors were not involved in the design of the study; the collection, analysis, and interpretation of data; writing the report; and did not impose any restrictions regarding the publication of the report.

Authors' relationships and activities AA is currently employed by The Novo Nordisk Foundation to establish a National Centre for Healthy Weight and is a member of the advisory board/consultant for Gelesis (USA), Groupe Éthique et Santé (France) and Weight Watchers (USA). AA is co-owner of the University of Copenhagen spin-off Flax-Slim ApS and is co-inventor on a pending provisional patent application for the use of biomarkers to predict responses to weight-loss diets and other related patents and patent applications that are all owned by the University of Copenhagen in accordance with Danish law. AA is co-author of a number of diet and cookery books, including books on personalised diet. AA is not an advocate or activist for specific diets and is not strongly committed to any specific diet (e.g. veganism, Atkins diet, gluten-free diet, high animal protein diet or dietary supplements). TML is an advisor for the 'Sense' diet programme. The remaining authors declare that there are no relationships or activities that might bias, or be perceived to bias, their work.

Contribution statement MNT participated in planning and conducting the study, including producing and analysing data, and writing the manuscript. MJS and AS assisted with planning of the study and data interpretation. MHC and PW contributed to production of data. AA, JJH, SM, JF and HST contributed to the conception and the design of the study and contributed to data production and interpretation. TML and FM contributed to the study design, supervised the food production and distribution, and assisted in data interpretation. $\mathrm{EC}, \mathrm{MF}$ and $\mathrm{BH}$ contributed to production and interpretation of data. TK and SBH conceptualised the study, obtained funding, supervised the study and contributed to interpretation of data. All authors participated in critical revision of the manuscript and approved the version to be published. MNT, TK and SBH are guarantors of this manuscript and, as such, take responsibility for the integrity of the data and the accuracy of the data analysis.

\section{References}

1. Kahn SE, Cooper ME, Del Prato S (2014) Pathophysiology and treatment of type 2 diabetes: perspectives on the past, present, and future. Lancet 383(9922):1068-1083. https://doi.org/10.1016/ S0140-6736(13)62154-6

2. Lean MEJ, Leslie WS, Barnes AC et al (2019) Durability of a primary care-led weight-management intervention for remission of type 2 diabetes: 2-year results of the DiRECT open-label, cluster-randomised trial. Lancet Diabetes Endocrinol 7(5):344355. https://doi.org/10.1016/S2213-8587(19)30068-3

3. Franz MJ, Boucher JL, Rutten-Ramos S, VanWormer JJ (2015) Lifestyle weight-loss intervention outcomes in overweight and obese adults with type 2 diabetes: a systematic review and metaanalysis of randomized clinical trials. J Acad Nutr Diet 115(9): 1447-1463. https://doi.org/10.1016/j.jand.2015.02.031

4. Heller SR, Group AC (2009) A summary of the ADVANCE trial. Diabetes Care 32(Suppl 2):S357-S361. https://doi.org/10.2337/ dc09-S339

5. Baum A, Scarpa J, Bruzelius E, Tamler R, Basu S, Faghmous J (2017) Targeting weight loss interventions to reduce cardiovascular complications of type 2 diabetes: a machine learning-based posthoc analysis of heterogeneous treatment effects in the Look AHEAD trial. Lancet Diabetes Endocrinol 5(10):808-815. https:// doi.org/10.1016/S2213-8587(17)30176-6

6. American Diabetes Association (2020) 5. Facilitating behavior change and well-being to improve health outcomes: standards of medical Care in Diabetes-2020. Diabetes Care 43(Suppl 1):S48S65. https://doi.org/10.2337/dc20-S005

7. Mann JI, De Leeuw I, Hermansen K et al (2004) Evidence-based nutritional approaches to the treatment and prevention of diabetes mellitus. Nutr Metab Cardiovasc Dis 14(6):373-394. https://doi. org/10.1016/s0939-4753(04)80028-0

8. Evert AB, Dennison M, Gardner CD et al (2019) Nutrition therapy for adults with diabetes or prediabetes: a consensus report. Diabetes Care 42(5):731-754. https://doi.org/10.2337/dci19-0014

9. Huntriss R, Campbell M, Bedwell C (2018) The interpretation and effect of a low-carbohydrate diet in the management of type 2 diabetes: a systematic review and meta-analysis of randomised controlled trials. Eur J Clin Nutr 72(3):311-325. https://doi.org/ 10.1038/s41430-017-0019-4

10. Skytte MJ, Samkani A, Petersen AD et al (2019) A carbohydratereduced high-protein diet improves $\mathrm{HbA} 1 \mathrm{c}$ and liver fat content in weight stable participants with type 2 diabetes: a randomised controlled trial. Diabetologia 62(11):2066-2078. https://doi.org/ 10.1007/s00125-019-4956-4

11. Mardinoglu A, Wu H, Bjornson E et al (2018) An integrated understanding of the rapid metabolic benefits of a carbohydrate-restricted diet on hepatic steatosis in humans. Cell Metab 27(3):559-571 e555. https://doi.org/10.1016/j.cmet.2018.01.005

12. Look ARG, Wing RR, Bolin P et al (2013) Cardiovascular effects of intensive lifestyle intervention in type 2 diabetes. N Engl J Med 369(2):145-154. https://doi.org/10.1056/NEJMoa1212914

13. Mifflin MD, St Jeor ST, Hill LA, Scott BJ, Daugherty SA, Koh YO (1990) A new predictive equation for resting energy expenditure in healthy individuals. Am J Clin Nutr 51(2):241-247. https://doi.org/ 10.1093/ajcn/51.2.241

14. Hall KD (2008) What is the required energy deficit per unit weight loss? Int J Obes 32(3):573-576. https://doi.org/10.1038/sj.ijo.0803720

15. Craig CL, Marshall AL, Sjostrom M et al (2003) International physical activity questionnaire: 12 -country reliability and validity. Med Sci Sports Exerc 35(8):1381-1395. https://doi.org/10.1249/ 01.MSS.0000078924.61453.FB

16. Carlsson ER, Fenger M, Henriksen T et al (2020) Reduction of oxidative stress on DNA and RNA in obese patients after roux- 
en-Y gastric bypass surgery-an observational cohort study of changes in urinary markers. PLoS One 15(12):e0243918. https:// doi.org/10.1371/journal.pone.0243918

17. Chabanova E, Fonvig CE, Bojsoe C, Holm JC, Thomsen HS (2017) (1)H MRS assessment of hepatic fat content: comparison between Normal- and excess-weight children and adolescents. Acad Radiol 24(8):982-987. https://doi.org/10.1016/j.acra.2017.02.010

18. Fonvig CE, Bille DS, Chabanova E, Nielsen TR, Thomsen HS, Holm JC (2012) Muscle fat content and abdominal adipose tissue distribution investigated by magnetic resonance spectroscopy and imaging in obese children and youths. Pediatr Rep 4(1):e11. https:// doi.org/10.4081/pr.2012.e11

19. Battelino T, Danne T, Bergenstal RM et al (2019) Clinical targets for continuous glucose monitoring data interpretation: recommendations from the international consensus on time in range. Diabetes Care 42(8):1593-1603. https://doi.org/10.2337/dci19-0028

20. Bingham SA (2003) Urine nitrogen as a biomarker for the validation of dietary protein intake. J Nutr 133 Suppl 3(3):921S-924S. https://doi.org/10.1093/jn/133.3.921S

21. American Diabetes Association (2020) 6. Glycemic targets: standards of medical Care in Diabetes-2020. Diabetes Care 43(Suppl 1):S66-S76. https://doi.org/10.2337/dc20-S006

22. Qian F, Korat AA, Malik V, Hu FB (2016) Metabolic effects of monounsaturated fatty acid-enriched diets compared with carbohydrate or polyunsaturated fatty acid-enriched diets in patients with type 2 diabetes: a systematic review and Meta-analysis of randomized controlled trials. Diabetes Care 39(8):1448-1457. https://doi. org $/ 10.2337 /$ dc $16-0513$

23. Nuttall FQ, Almokayyad RM, Gannon MC (2015) Comparison of a carbohydrate-free diet vs. fasting on plasma glucose, insulin and glucagon in type 2 diabetes. Metabolism 64(2):253-262. https:// doi.org/10.1016/j.metabol.2014.10.004

24. Samkani A, Skytte MJ, Kandel D et al (2018) A carbohydratereduced high-protein diet acutely decreases postprandial and diurnal glucose excursions in type 2 diabetes patients. Br J Nutr 119(8): 910-917. https://doi.org/10.1017/S0007114518000521

25. Cavalot F, Petrelli A, Traversa M et al (2006) Postprandial blood glucose is a stronger predictor of cardiovascular events than fasting blood glucose in type 2 diabetes mellitus, particularly in women: lessons from the san Luigi Gonzaga diabetes study. J Clin Endocrinol Metab 91(3):813-819. https://doi.org/10.1210/jc.2005-1005

26. Thomsen MN, Skytte MJ, Astrup A et al (2020) The clinical effects of a carbohydrate-reduced high-protein diet on glycaemic variability in metformin-treated patients with type 2 diabetes mellitus: a randomised controlled study. Clin Nutr ESPEN 39:46-52. https:// doi.org/10.1016/j.clnesp.2020.07.002

27. Tay J, Thompson CH, Luscombe-Marsh ND et al (2018) Effects of an energy-restricted low-carbohydrate, high unsaturated fat/low saturated fat diet versus a high-carbohydrate, low-fat diet in type 2 diabetes: a 2-year randomized clinical trial. Diabetes Obes Metab 20(4):858-871. https://doi.org/10.1111/dom.13164

28. Kotronen A, Juurinen L, Hakkarainen A et al (2008) Liver fat is increased in type 2 diabetic patients and underestimated by serum alanine aminotransferase compared with equally obese nondiabetic subjects. Diabetes Care 31(1):165-169. https://doi.org/10.2337/ dc07-1463

29. Donnelly KL, Smith CI, Schwarzenberg SJ, Jessurun J, Boldt MD, Parks EJ (2005) Sources of fatty acids stored in liver and secreted via lipoproteins in patients with nonalcoholic fatty liver disease. J Clin Invest 115(5):1343-1351. https://doi.org/10.1172/JCI23621

30. Schwarz JM, Linfoot P, Dare D, Aghajanian K (2003) Hepatic de novo lipogenesis in normoinsulinemic and hyperinsulinemic subjects consuming high-fat, low-carbohydrate and low-fat, high- carbohydrate isoenergetic diets. Am J Clin Nutr 77(1):43-50. https://doi.org/10.1093/ajcn/77.1.43

31. Bozzetto L, Prinster A, Annuzzi G et al (2012) Liver fat is reduced by an isoenergetic MUFA diet in a controlled randomized study in type 2 diabetic patients. Diabetes Care 35(7):1429-1435. https:// doi.org/10.2337/dc12-0033

32. Sandby K, Geiker NRW, Dalamaga M, Gronbaek H, Magkos F (2021) Efficacy of dietary manipulations for depleting intrahepatic triglyceride content: implications for the Management of nonalcoholic Fatty Liver Disease. Curr Obes Rep 10(2):125-133. https://doi.org/10.1007/s13679-021-00430-4

33. Kirk E, Reeds DN, Finck BN, Mayurranjan SM, Patterson BW, Klein S (2009) Dietary fat and carbohydrates differentially alter insulin sensitivity during caloric restriction. Gastroenterology 136(5):1552-1560. https://doi.org/10.1053/j.gastro.2009.01.048

34. Haufe S, Engeli S, Kast P et al (2011) Randomized comparison of reduced fat and reduced carbohydrate hypocaloric diets on intrahepatic fat in overweight and obese human subjects. Hepatology 53(5):1504-1514. https://doi.org/10.1002/hep.24242

35. Inaishi J, Saisho Y (2020) Beta-cell mass in obesity and type 2 diabetes, and its relation to pancreas fat: a Mini-review. Nutrients 12(12):3846. https://doi.org/10.3390/nu12123846

36. Al-Mrabeh A, Hollingsworth KG, Shaw JAM et al (2020) 2-year remission of type 2 diabetes and pancreas morphology: a post-hoc analysis of the DiRECT open-label, cluster-randomised trial. Lancet Diabetes Endocrinol 8(12):939-948. https://doi.org/10. 1016/S2213-8587(20)30303-X

37. Kirkpatrick CF, Bolick JP, Kris-Etherton PM et al (2019) Review of current evidence and clinical recommendations on the effects of low-carbohydrate and very-low-carbohydrate (including ketogenic) diets for the management of body weight and other cardiometabolic risk factors: a scientific statement from the National Lipid Association Nutrition and lifestyle task force. J Clin Lipidol 13(5):689-711 e681. https://doi.org/10.1016/j.jacl.2019.08.003

38. Nezu U, Kamiyama H, Kondo Y, Sakuma M, Morimoto T, Ueda S (2013) Effect of low-protein diet on kidney function in diabetic nephropathy: meta-analysis of randomised controlled trials. BMJ Open 3(5):e002934. https://doi.org/10.1136/bmjopen-2013-002934

39. Goldenberg JZ, Day A, Brinkworth GD et al (2021) Efficacy and safety of low and very low carbohydrate diets for type 2 diabetes remission: systematic review and meta-analysis of published and unpublished randomized trial data. BMJ 372:m4743. https://doi. org/10.1136/bmj.m4743

40. Gannon MC, Hoover H, Nuttall FQ (2010) Further decrease in glycated hemoglobin following ingestion of a LoBAG30 diet for 10 weeks compared to 5 weeks in people with untreated type 2 diabetes. Nutr Metab (Lond) 7:64. https://doi.org/10.1186/1743-7075-7-64

41. Silva FM, Kramer CK, de Almeida JC, Steemburgo T, Gross JL, Azevedo MJ (2013) Fiber intake and glycemic control in patients with type 2 diabetes mellitus: a systematic review with metaanalysis of randomized controlled trials. Nutr Rev 71(12):790801. https://doi.org/10.1111/nure.12076

42. Schwingshackl L, Strasser B, Hoffmann G (2011) Effects of monounsaturated fatty acids on glycaemic control in patients with abnormal glucose metabolism: a systematic review and meta-analysis. Ann Nutr Metab 58(4):290-296. https://doi.org/10.1159/000331214

43. Alzahrani AH, Skytte MJ, Samkani A et al (2021) Body weight and metabolic risk factors in patients with type 2 diabetes on a selfselected high-protein low-carbohydrate diet. Eur J Nutr 60(8): 4473-4482. https://doi.org/10.1007/s00394-021-02605-0

Publisher's note Springer Nature remains neutral with regard to jurisdictional claims in published maps and institutional affiliations. 